Pacific Journal of Mathematics

EIGENVALUE BOUNDS FOR THE DIRAC OPERATOR 


\section{EIGENVALUE BOUNDS FOR THE DIRAC OPERATOR}

\section{JOHN LOTT}

A natural question in the study of geometric operators is that of how much information is needed to estimate the eigenvalues of an operator. For the square of the Dirac operator, such a question has at least peripheral physical import. When coupled to gauge fields, the lowest eigenvalue is related to chiral symmetry breaking. In the pure metric case, lower eigenvalue estimates may help to give a sharper estimate of the ADM mass of an asymptotically flat spacetime with black holes. We use three tools to estimate the eigenvalues of the square of the (purely metric) Dirac operator: the conformal covariance of the operator, a patching method and a heat kernel bound.

I. A lower bound. Let $V$ be a vector bundle associated to the $\operatorname{SO}(n)$ $(\operatorname{Spin}(n))$ frame bundle of a compact $n$-dimensional oriented (spin) Riemannian manifold $X$, with a positive-definite inner product $\langle$,$\rangle . For$ each metric $g$, let $T_{g}: C^{\infty}(V) \rightarrow C^{\infty}(V)$ be a geometric elliptic symmetric differential operator of order $j<n$. If $g^{\prime}=e^{2 \sigma} g$ is a conformally related metric, suppose that $T_{g^{\prime}}=e^{-j \sigma} e^{-(n-j) \sigma / 2} T_{g} e^{(n-j) \sigma / 2}$. Let $\lambda_{1}^{2}(g)$ denote the lowest eigenvalue of $T_{g}^{2}$.

Proposition 1. (i) If $T_{g}$ is invertible then $\exists c>0$ s.t. $\forall g^{\prime} \in[g]$, (the conformal class of $g$ ),

$$
\lambda_{1}^{2}\left(g^{\prime}\right) \geq c^{-2}\left(\operatorname{Vol} g^{\prime}\right)^{-2 j / n} .
$$

(ii) Suppose that a multiple $m V$ of $V$ contains a trivial subbundle of real dimension $>n$. Then the best constant $\tilde{c}$ in (1) is

$$
d \equiv \sup _{f \neq 0}\left|\int\left\langle f, T_{g}^{-1} f\right\rangle d \operatorname{vol}\right| /\|f\|_{2 n /(n+j)}^{2} .
$$

Proof. (i) Let $\psi$ range through $C^{\infty}(V)$. Then

$$
\begin{aligned}
& \lambda_{1}^{-1}\left(g^{\prime}\right)=\sup _{\psi \neq 0}\left|\int\left\langle\psi, T_{g^{\prime}}^{-1} \psi\right\rangle d \mathrm{vol}^{\prime}\right| / \int\langle\psi, \psi\rangle d \mathrm{vol}^{\prime} \\
& =\sup _{\psi \neq 0} \mid \int e^{n \sigma}\left\langle\psi, e^{-(n-j) \sigma / 2} T_{g}^{-1} e^{(n+j) \sigma / 2} \psi\right\rangle d \text { vol } \mid / \int e^{n \sigma}\langle\psi, \psi\rangle d \text { vol } \\
& =\sup _{f \neq 0} \mid \int\left\langle f, T_{g}^{-1} f\right\rangle d \text { vol } \mid / \int e^{-j \sigma}\langle f, f\rangle d \text { vol. }
\end{aligned}
$$


By Hölder's inequality,

Then

$$
\left(\int|f|^{2 n /(n+j)} d \mathrm{vol}\right)^{(n+j) / n} \leq\left(\int e^{-j \sigma}|f|^{2} d \mathrm{vol}\right)\left(\mathrm{Vol} g^{\prime}\right)^{j / n}
$$

$$
\begin{aligned}
\lambda_{1}^{-1}\left(g^{\prime}\right)\left(\operatorname{Vol} g^{\prime}\right)^{j / n} & \leq \sup _{f \neq 0}\left|\int\left\langle f, T_{g}^{-1} f\right\rangle d \operatorname{vol}\right| /\left(\int|f|^{2 n /(n+j)} d \text { vol }\right)^{(n+j) / n} \\
& \leq \sup _{f \neq 0}\left(\|f\|_{2} /\left\|\left|T_{g}\right|^{1 / 2} f\right\|_{2 n /(n+j)}\right)^{2} .
\end{aligned}
$$

Because $\left(I+\nabla^{+} \nabla\right)^{j / 4}\left|T_{g}\right|^{-1 / 2}$ is bounded on $L_{2 n /(n+j)}$ and $\mathscr{L}_{2 n /(n+j)}^{j / 2} \hookrightarrow$ $L_{2}$, [5], the RHS is finite.

(ii) Consider $T_{g^{\prime}}=m T_{g^{\prime}}$ acting on $m V$ with a $C^{\infty}$ section $\tilde{\psi}$. Then

$$
\begin{aligned}
\tilde{c} & =\sup _{\tilde{\psi} \neq 0}\left|\int\left\langle\tilde{\psi}, \tilde{T}_{g}^{-1} \tilde{\psi}\right\rangle d \operatorname{vol}^{\prime}\right| /\left(\operatorname{Vol}_{g^{\prime}}\right)^{j / n}\left(\int\langle\tilde{\psi}, \tilde{\psi}\rangle d \mathrm{vol}^{\prime}\right) \\
& \leq \sup _{\tilde{f} \neq 0}\left|\int\left\langle\tilde{f}, \tilde{T}_{g}^{-1} \tilde{f}\right\rangle d \operatorname{vol}\right| /\|\tilde{f}\|_{2 n /(n+j)}^{2}=d .
\end{aligned}
$$

With the hypothesis, the generic section of $m V$ has no zeroes. Let $\left\{\tilde{f}_{i}\right\}$ be a sequence in $C^{\infty}(m V)$ approaching the sup $d$. By perturbing each $\tilde{f}_{i}$ arbitrarily little in the $C^{\infty}$ topology, we can assume that each $\tilde{f}_{i}$ has no zeroes. Define $g_{i}^{\prime}=\left|\tilde{f}_{i}\right|^{4 /(n+j)} g$ and $\tilde{\psi}_{i}=\tilde{f}_{i} /\left|\tilde{f}_{i}\right|$. Then

$$
\begin{aligned}
d & =\lim _{i}\left|\int\left\langle\tilde{f}_{i}, \tilde{T}_{g}^{-1} \tilde{f}_{i}\right\rangle d \operatorname{vol}\right| /\left\|\tilde{f}_{i}\right\|_{2 n /(n+j)}^{2} \\
& =\lim _{i}\left|\int\left\langle\tilde{\psi}_{i}, \tilde{T}_{g_{i}^{\prime}}^{-1} \tilde{\psi}_{i}\right\rangle d \operatorname{vol}_{i}^{\prime}\right| /\left(\left(\int\left\langle\tilde{\psi}_{i}, \tilde{\psi}_{i}\right\rangle d \operatorname{vol}_{i}^{\prime}\right)\left(\operatorname{Vol}_{i}^{\prime}\right)^{j / n}\right) \leq \tilde{c}
\end{aligned}
$$

II. The Dirac operator. For background on the Dirac operator we refer to [4]. $X$ is a spin manifold with a fixed spin structure. The spinor bundle $V$ is associated to the principal $\operatorname{Spin}(n)$ bundle over $X$. The Dirac operator is the composition $\boxplus: C^{\infty}(V) \stackrel{\nabla}{\rightarrow} C^{\infty}(V) \otimes \Lambda^{1}(X) \rightarrow C^{\infty}(V)$, the last map being Clifford multiplication.

Proposition 2. Take $\mathrm{g}^{\prime}=e^{2 \sigma} \mathrm{g}$. Then

$$
\bigoplus_{g^{\prime}}=e^{-\sigma} e^{-(n-1) \sigma / 2} \bigoplus_{g} e^{(n-1) \sigma / 2} \text {. }
$$

Proof. Let $\left\{e_{j}\right\}_{j=1}^{n}$ be an orthonormal frame for $g$, with dual frame $\left\{\tau_{j}\right\}_{j=1}^{n}$. Locally, $\bigoplus_{g}=-i \sum_{i=1}^{n} \gamma^{j} \nabla_{e_{j}}$, with $\left\{\gamma_{j}\right\}_{j=1}^{n} \in \operatorname{End}\left(C^{2^{[n / 2]}}\right)$ satisfying $\left\{\gamma^{i}, \gamma^{j}\right\}=2 \delta^{i j}$ and $\nabla_{e_{j}}=e_{j}+\frac{1}{4}\left\langle\omega_{a b}, e_{j}\right\rangle \gamma^{a} \gamma^{b}$. The new orthonormal 
frame for $g^{\prime}$ is $\left\{e_{j}^{\prime}\right\}_{j=1}^{n}=\left\{e^{-\sigma} e_{j}\right\}_{j=1}^{n}$. The new connection is $\omega_{a b}^{\prime}=\omega_{a b}$ $\left(e_{a} \sigma\right) \tau_{b}+\left(e_{b} \sigma\right) \tau_{a}$. Then

$$
\begin{aligned}
\bigoplus_{g^{\prime}} & =-i \sum \gamma^{j} \nabla_{e^{\prime},}=-i \sum \gamma^{j}\left(e_{j}^{\prime}+\frac{1}{4}\left\langle\omega_{a b}^{\prime}, e_{j}^{\prime}\right\rangle \gamma^{a} \gamma^{b}\right) \\
& =-i e^{-\sigma} \sum \gamma^{j}\left(\nabla_{j}+\frac{1}{4}\left(e_{b} \sigma\right)\left[\gamma^{j}, \gamma^{b}\right]\right) \\
& =-i e^{-\sigma} \sum \gamma^{j}\left(\nabla_{j}+\frac{n-1}{2} e_{j} \sigma\right)=e^{-\sigma} e^{-(n-1) \sigma / 2} \bigoplus_{g} e^{(n-1) \sigma / 2}
\end{aligned}
$$

Thus $\boxplus$ is conformally covariant with $j=1$. This differs from the corresponding equation in [4], which has an additional line bundle tensored, by the factor $e^{-\sigma}$, but does not change the conclusion of [4] that the dimension of the harmonic spinor space is conformally invariant. The two Dirac operators can be compared because the conformal change in the metric does not affect the spinor bundle; only the soldering form on the $\operatorname{Spin}(n)$ bundle is changed, not the bundle itself.

Equation (1) implies, in particular, that on $S^{2}, \exists c>0$ s.t. $\forall g$, $\lambda_{1}^{2}(g) \geq c^{-2}(\mathrm{Vol} g)^{-1}$. On the standard $S^{2}, \lambda_{1}=1$. Thus the best constant $d$ satisfies $d \geq 1 / \sqrt{4 \pi}$. It appears that $d=1 / \sqrt{4 \pi}$, although we have no proof.

The conformal covariance can also be used to get upper bounds on $\lambda_{1}^{2}$

Proposition 3. Given a conformal glass $[g], \exists b>0$ s.t. $\forall g^{\prime} \in[g]$ with $R\left(g^{\prime}\right)<0, \lambda_{1}^{2}\left(g^{\prime}\right) \leq-b R_{\min }\left(g^{\prime}\right)$.

Proof. Fix a $g$ in the conformal class s.t. $R(g)<0$ and write $g^{\prime}=e^{2 \sigma} g$. For any $\psi \in C^{\infty}(V)$,

$$
\begin{aligned}
\lambda_{1}^{2}(g) & \leq \int e^{n \sigma}\left|\bigoplus_{g^{\prime}} \psi\right|^{2} d \mathrm{vol} / \int e^{n \sigma}|\psi|^{2} d \text { vol } \\
& =\int e^{-\sigma}\left|\bigoplus_{g} e^{(n-1) \sigma / 2} \psi\right|^{2} d \mathrm{vol} / \int e^{\sigma}\left|e^{(n-1 / 2) \sigma} \psi\right|^{2} d \text { vol. }
\end{aligned}
$$

Take $e^{(n-1 / 2) \sigma} \psi=\psi_{0}$, a lowest eigenfunction of $\boxplus_{g}$. Then

$$
\lambda_{1}^{2}\left(g^{\prime}\right) \leq \lambda_{1}^{2}(g)\left(\sup \left|\psi_{0}\right|^{2} / \mathrm{inf}\left|\psi_{0}\right|^{2}\right) \int e^{-\sigma} d \mathrm{vol} / \int e^{\sigma} d \text { vol. }
$$

For $n \geq 3$,

$$
-4 \frac{n-1}{n-2} e^{-n \sigma / 2} \nabla^{2} e^{(n-2) \sigma / 2}+R(g) e^{-\sigma}=R\left(g^{\prime}\right) e^{\sigma} .
$$


Then

$$
\begin{aligned}
& \left(R_{\max }(g) / R_{\min }\left(g^{\prime}\right)\right)\left(\int e^{-\sigma} d \mathrm{vol} / \int e^{\sigma} d \mathrm{vol}\right) \\
& \quad \leq \int R(g) e^{-\sigma} d \mathrm{vol} / \int R\left(g^{\prime}\right) e^{\sigma} d \mathrm{vol} \\
& \quad=1+n(n-1) \int e^{-\sigma}|\nabla \sigma|^{2} d \mathrm{vol} / \int R\left(g^{\prime}\right) e^{\sigma} d \mathrm{vol} \leq 1
\end{aligned}
$$

and

$$
\lambda_{1}^{2}\left(g^{\prime}\right) \leq \lambda_{1}^{2}(g)\left(\sup \left|\psi_{0}\right|^{2} / \inf \left|\psi_{0}\right|^{2}\right)\left(R_{\min }\left(g^{\prime}\right) / R_{\max }(g)\right) .
$$

For $n=2,-e^{-\sigma} \nabla^{2} \sigma+R(g) e^{-\sigma}=R\left(g^{\prime}\right) e^{\sigma}$,

$$
\begin{aligned}
& \int R e^{-\sigma} d \text { vol } / \int R\left(g^{\prime}\right) e^{\sigma} d \text { vol } \\
& \quad=1+\int e^{-\sigma}|\nabla \sigma|^{2} d \text { vol } / \int R\left(g^{\prime}\right) e^{\sigma} d \text { vol } \leq 1
\end{aligned}
$$

and the same result holds.

III. A patching method. We give an upper bound on $S^{2}$ using the method of [1].

Proposition 4. Let $M_{l}$ be the set of metrics on $S^{2}$ with Gaussian curvature $K$ satisfying $0 \leq K \leq l$. Then $\exists \alpha>0$ s.t. $\forall l \in R^{+}$and $\forall g \in M_{l}$, $\lambda_{1}^{2}(g) \leq \alpha l$.

Proof. First we solve for the lowest eigenfunction of the Dirichlet problem for $\boxplus^{2}$ on the unit disk. Take

$$
\gamma^{r}=\left(\begin{array}{ll}
0 & 1 \\
1 & 0
\end{array}\right), \quad \gamma^{\theta}=\left(\begin{array}{cc}
0 & -i \\
i & 0
\end{array}\right), \quad e_{r}=\frac{\partial}{\partial r} \quad \text { and } \quad e_{\theta}=\frac{1}{r} \frac{\partial}{\partial \theta}
$$

Then

$$
\bigoplus^{2}=-\left(e_{r}+\frac{1}{2 r}\right)^{2}-e_{\theta}^{2}+\gamma^{r} \gamma^{\theta} \frac{1}{r} e_{\theta}
$$

Take

$$
\begin{aligned}
\psi=\left(\eta_{1}(r) e^{i\left(m_{1}+1 / 2\right) \theta}, \eta_{2}(r) e^{i\left(m_{2}-1 / 2\right) \theta}\right), & \\
\eta_{1}(1)=\eta_{2}(1)=0, m_{1}, m_{2} & \in Z .
\end{aligned}
$$


Then

$$
\begin{aligned}
\bigoplus^{2} \psi=\left(-\left(\frac{\partial}{\partial r}+\frac{1}{2 r}\right)^{2}\right. & \eta_{1}+\frac{1}{r^{2}}\left(m_{1}^{2}-\frac{1}{4}\right) \eta_{1} \\
& \left.-\left(\frac{\partial}{\partial r}+\frac{1}{2 r}\right)^{2} \eta_{2}+\frac{1}{r^{2}}\left(m_{2}^{2}-\frac{1}{4}\right) \eta_{2}\right) .
\end{aligned}
$$

WLOG, we can assume $\eta_{2}=0$. The lowest eigenfunction is $\psi_{0}=$ $\left(J_{0}(z r) e^{\imath \theta / 2}, 0\right)$ with eigenvalue $\lambda_{1}^{2}=z^{2}, z$ being the first zero of $J_{0}$.

Take normal coordinates around a point $x$ in $S^{2}$ and write $g$ as $d r^{2}+f(r, \theta) d \theta^{2}$. With $e_{r}=\partial / \partial r$ and $e_{\theta}=f^{-1 / 2} \partial / \partial \theta$,

$$
\boldsymbol{D}_{g}=-i \gamma^{r}\left(e_{r}+\frac{1}{4 f} f,_{r}\right)-i \gamma^{\theta} e_{\theta} \text {. }
$$

WLOG, we can assume $l=\pi^{2}$. Put $D=\left\{y \in S^{2}\right.$ : $\exists$ ! minimal geodesic from $x$ to $y$ and $d(x, y)<1\}$, a contractible domain. We wish to patch the Dirichlet solution onto $X$. Define $\psi \in L^{2}(V)$ by

$$
\psi(y)= \begin{cases}\left(J_{0}(z d(x, y)) e^{i \theta / 2}, 0\right) & \text { if } y \in D, \\ 0 & \text { if } y \notin D .\end{cases}
$$

Then $\psi$ is $C^{1}$ a.e. and

$$
\begin{aligned}
\lambda_{1}^{2} & \leq \int|\Phi \psi|^{2} d \text { vol } / \int|\psi|^{2} d \text { vol } \\
& =\frac{\int_{S_{1}} \int_{0}^{a(\theta)} f^{1 / 2}\left(\partial_{r} J_{0}(z r)+\left(\frac{1}{4 f} f,_{r}-\frac{1}{2 r}\right) J_{0}(z r)\right)^{2} d r d \theta}{\int_{S^{1}} \int_{0}^{a(\theta)} f^{1 / 2} J_{0}^{2}(z r) d r d \theta},
\end{aligned}
$$

where $a(\theta)$ is $\min ($ distance to the cut locus of $x$ along angle $\theta, 1$ ). Now

$$
\begin{aligned}
\int_{0}^{a(\theta)} & f^{1 / 2}\left(\partial_{r} J_{0}(z r)+\left(\frac{1}{4 f} f,_{r}-\frac{1}{2 r}\right) J_{0}(z r)\right)^{2} d r \\
= & \int_{0}^{a(\theta)} f^{1 / 2}\left(r^{1 / 2} f^{-1 / 4} \partial_{r}\left(r^{-1 / 2} f^{1 / 4} J_{0}(z r)\right)\right)^{2} d r \\
= & {\left[r^{1 / 2} f^{1 / 4} J_{0}(z r) \partial_{r}\left(r^{-1 / 2} f^{1 / 4} J_{0}(z r)\right)\right]_{r=0}^{a(\theta)} } \\
& \quad-\int_{0}^{a(\theta)} f^{1 / 2} J_{0}(z r)\left(r^{-1 / 2} f^{-1 / 4} \partial_{r}\left(r \partial_{r}\left(r^{-1 / 2} f^{1 / 4} J_{0}(z r)\right)\right)\right) d r \\
= & {[\partial \text { term }]-\int_{0}^{a(\theta)} f^{1 / 2} J_{0}(z r)\left[\partial_{r} \partial_{r} J_{0}(z r)+\frac{1}{2} f,_{r} f^{-1} \partial_{r} J_{0}(z r)\right.} \\
& \left.\quad+\left(\frac{1}{4 r^{2}}-\frac{3}{16}\left(f,{ }_{r}\right)^{2} f^{-2}+\frac{1}{4} f,,_{r r} f^{-1}\right) J_{0}(z r)\right] d r .
\end{aligned}
$$


Put $\nabla_{\partial_{r}} \partial_{\theta} \equiv c \partial_{\theta}$. Then $f_{,_{r}}=2 c f$ and $f_{, r}=2\left(c^{2}-K\right) f$. By Rauch's comparison theorem, there are no conjugate points in $D$ and $\left(1 / \pi^{2}\right) \operatorname{Sin}^{2} \pi r \leq$ $f \leq r^{2}, \pi \operatorname{Cot} \pi r \leq c \leq 1 / r$ in $D$. Thus [ $\partial$ term] $\leq 0$ and

$$
\begin{gathered}
-\frac{1}{\int_{0}^{a(\theta)} f^{1 / 2} J_{0}^{2}(z r) d r} \\
\cdot \int_{0}^{a(\theta)} f^{1 / 2} J_{0}(z r)\left(\partial_{r} \partial_{r} J_{0}(z r)+\frac{1}{2} f, f_{r}{ }^{-1} \partial_{r} J_{0}(z r)\right. \\
\left.+\left(\frac{1}{4 r^{2}}-\frac{3}{16}\left(f,_{r}\right)^{2} f^{-2}+\frac{1}{4} f,_{r r} f^{-1}\right)\left(J_{0}(z r)\right)\right) d r \\
\left.\leq-\frac{1}{\int_{0}^{a(\theta)} f^{1 / 2} J_{0}^{2}(z r) d r} \quad+\frac{1}{r} \partial_{r} J_{0}(z r)+\left(\frac{1}{4 r^{2}}-\frac{1}{4} c^{2}-\frac{1}{2} K\right) J_{0}(z r)\right) d r \\
\cdot \int_{0}^{a(\theta)} f^{1 / 2} J_{0}(z r)\left(\partial_{r} \partial_{r} J_{0}(z r)\right. \\
=z^{2}+\int_{0}^{a(\theta)} f^{1 / 2} J_{0}^{2}(z r)\left(\frac{1}{4}\left(c^{2}-\frac{1}{r^{2}}\right)+\frac{1}{2} K\right) d r / \int_{0}^{a(\theta)} f^{1 / 2} J_{0}^{2}(z r) d r \\
\leq z^{2}+\frac{\pi^{2}}{2}+\frac{\frac{1}{4} \int_{0}^{a(\theta)} f^{1 / 2} J_{0}^{2}(z r)\left(\max \left(\pi^{2} \operatorname{Cot}^{2} \pi r, \frac{1}{r^{2}}\right)-\frac{1}{r^{2}}\right) d r}{\int_{0}^{a(\theta)} f^{1 / 2} J_{0}^{2}(z r) d r} \\
\frac{\pi^{2}}{2}+\frac{\frac{1}{4} \int_{0}^{a(\theta)} J_{0}^{2}(z r)\left[r \max \left(\pi^{2} \operatorname{Cot}^{2} \pi r, \frac{1}{r^{2}}\right)-\frac{1}{\pi}(\operatorname{Sin} \pi r) \frac{1}{r^{2}}\right] d r}{z^{2}\left(\operatorname{Sin}^{2}\right) J_{0}^{2}(z r) d r} .
\end{gathered}
$$

Thus $\lambda_{1}^{2} \leq \alpha l$ with

$\alpha=\frac{\frac{1}{\pi^{2}}\left[z^{2}+\frac{\pi^{2}}{2}+\frac{1}{4} \sup _{0 \leq a \leq 1} \int_{0}^{a} J_{0}^{2}(z r)\left[r \max \left(\pi^{2} \operatorname{Cot}^{2} \pi r, r^{-2}\right)-\pi^{-1}(\operatorname{Sin} \pi r) r^{-2}\right] d r\right]}{\int_{0}^{a} \frac{1}{\pi}(\operatorname{Sin} \pi r) J_{0}^{2}(z r) d r}$

$<\infty$.

IV. Heat kernel estimates. The higher eigenvalues of the Dirac operator can be estimated from below via upper bounds on the heat kernel of $\boldsymbol{\oplus}^{2}$. 
Proposition 5. If $n>1$ then

$\forall \alpha>0, j e^{-\alpha} \leq 2^{[n / 2]} \int_{X} e^{-\left(\alpha / 4 \lambda_{\jmath}\right) R(x)}\left(\operatorname{Vol}(X)^{-1}+4\left(\frac{2 C_{1}}{n} \frac{\alpha}{\lambda_{j}}\right)^{-n / 2}\right)$

with

$$
C_{1}=\inf _{\substack{\int f=0 \\ f \in H_{1}(x)}} \int|\nabla f|^{2} /\left(\int f^{2}\right)^{(2+n / n)}\left(\int|f|\right)^{-4 / n} .
$$

Proof. We have that both $\boxplus=\nabla^{+} \nabla+R / 4$ and $R / 4$ are self-adjoint on the unique closed extension of ${\boldsymbol{D}^{2}}_{C^{\infty}(V)}$ [7]. By the GoldenThomson inequality,

$$
\operatorname{Tr} e^{-T \nexists^{2}} \leq \operatorname{Tr} e^{-T R / 8} e^{-T \nabla^{+} \nabla} e^{-T R / 8}=\int_{X} e^{-T R(x) / 4} \tau\left(e^{-T \nabla^{+} \nabla}\right)(x, x),
$$

with $\tau$ being the local fiber trace.

We write $e^{-T \nabla^{+}} \nabla(x, x)$ as a Feynman-Kac path integral. This is given as a limit of approximations, each of which can be estimated.

Let $r$ be the cut radius of $X, K_{T}(x, y)$ be the kernel of $e^{-T \Delta}$ and $\rho(a)$ be a bump function which is 1 near 0 and 0 for $|a|>r / 2$. Define the operator $L_{T}$ on $L^{2}(V)$ by

$$
L_{T}(x, y)=K_{T}(x, y)\left(P \exp \left\{-\int_{y}^{x} \frac{1}{2} \omega_{a b} \sigma^{a b}\right\}\right) \rho(d(x, y)),
$$

the path-ordered integral being taken along the unique minimal geodesic from $x$ to $y$. Then for $\psi \in L^{2}(V)$,

$$
\begin{aligned}
\lim _{T \rightarrow 0} \frac{d}{d T} L_{T} \psi(x)=\lim _{T \rightarrow 0} \int\left(-\Delta_{y} K_{T}(x, y)\right) P \\
\quad \times \exp \left\{-\int_{y}^{x} \frac{1}{2} \omega_{a b} \sigma^{a b}\right\} \rho(d(x, y)) \psi(y) d y \\
=\lim _{T \rightarrow 0}-\int K_{T}(x, y) \Delta_{y}\left(P \exp \left\{-\int_{y}^{x} \frac{1}{2} \omega_{a b} \sigma^{a b}\right\} \rho(d(x, y)) \psi(y)\right) d y \\
=-\int \delta(x-y) \Delta_{y} P \exp \left\{-\int_{y}^{x} \frac{1}{2} \omega_{a b} \sigma^{a b}\right\} \psi(y) d y .
\end{aligned}
$$

Choose normal coordinates around $x$ and a synchronous frame $\tau^{i}$. Then

$$
P \exp \left\{-\int_{y}^{x} \frac{1}{2} \omega_{a b} \sigma^{a b}\right\}=1
$$


and

$$
-\left.\Delta_{y} P \exp \left\{-\int_{y}^{x} \frac{1}{2} \omega_{a b} \sigma^{a b}\right\} \psi(y)\right|_{y=x}=\partial^{2} \psi(x)=-\left(\nabla^{+} \nabla \psi\right)(x) .
$$

Thus

$$
\lim _{n \rightarrow \infty} n\left\|\left(e^{-T \nabla^{+} \nabla / n}-L_{T / n}\right) \psi\right\|=0
$$

and

$$
\begin{aligned}
& \left\|\left(e^{-T \nabla^{+} \nabla}-L_{T / n}^{n}\right) \psi\right\| \\
& \quad=\left\|\sum_{i=0}^{n-1} L_{T / n}^{i}\left(e^{-T / n \nabla^{+} \nabla}-L_{T / n}\right)\left(e^{-T / n \nabla^{+} \nabla}\right)^{n-i-1} \psi\right\| \| \\
& \quad \leq n \sup _{0 \leq s \leq T}\left\|\left(e^{-T / n \nabla^{+} \nabla}-L_{T / n}\right) e^{-s \nabla^{+} \nabla} \psi\right\| \rightarrow 0,
\end{aligned}
$$

showing that $e^{-T \nabla^{+} \nabla}=s \lim _{n \rightarrow \infty} L_{T / n}^{n}$.

Let $d \mu_{T, x, y}$ denote the Wiener measure on paths $\gamma$ going from $y$ to $x$ in time $T$. Then for $\psi, \eta \in L^{2}(V)$,

$$
\begin{aligned}
\left|\left\langle\psi,,^{-T \nabla^{+} \nabla} \eta\right\rangle\right|= & \lim _{n \rightarrow \infty}\left|\left\langle\psi, L_{T / n}^{n} \eta\right\rangle\right| \\
= & \lim _{n \rightarrow \infty}\left|\iint \psi^{+}(x) P \exp \left\{-\int_{\tilde{\gamma}_{n}} \frac{1}{2} \omega_{a b} \sigma^{a b}\right\} \eta(y)\right| \\
& \times \prod_{i=0}^{n-1} \rho\left(d\left(\gamma\left(\frac{i T}{n}\right), \gamma\left(\frac{(i+1) T}{n}\right)\right)\right) d \mu_{T, x, y}(\gamma) d x d y \mid,
\end{aligned}
$$

$\tilde{\gamma}_{n}$ being the broken geodesic connecting the points $\{\gamma(i T / n)\}_{i=0}^{n}$. Because $P \exp \left\{-\int_{\tilde{\gamma}_{n}} \frac{1}{2} \omega_{a b} \sigma^{a b}\right\}$ is in Spin, this is

$$
\leq \int|\psi(x)||\eta(y)| d \mu_{T, x, y}(\gamma) d x d y .
$$

Letting $\psi$ and $\eta$ approach $V$-valued $\delta$-functions with support at $x$ and values $\psi_{0}$ and $\eta_{0}$,

$$
\left\langle\psi_{0}, e^{-T \nabla^{+} \nabla}(x, x) \eta_{0}\right\rangle \leq\left|\psi_{0}\right|\left|\eta_{0}\right| \int d \mu_{T, x, y}(\gamma)=\left|\psi_{0}\right|\left|\eta_{0}\right| K_{T}(x, x) .
$$

Thus

$$
\tau\left(e^{-T \nabla^{+} \nabla}(x, x)\right) \leq(\operatorname{dim} V) K_{T}(x, x) .
$$


Now

$$
K_{T}(x, x) \leq \operatorname{vol}(X)^{-1}+4\left(2 C_{1} T / n\right)^{-n / 2} \quad[2]
$$

Thus

$$
\operatorname{Tr} e^{-t \oplus^{2}} \leq(\operatorname{dim} V) \int e^{-T R(x) / 4}\left[\operatorname{vol}(X)^{-1}+4\left(\frac{2 C_{1}}{n} T\right)^{-n / 2}\right]
$$

Putting $T=\alpha / \lambda_{j}$ gives the desired result.

COROLlaRY. For $j \geq 2^{[n / 2]} e^{n / 2}$,

$$
\lambda_{j} \geq c_{1}(4 \operatorname{Vol}(X))^{-2 / n}\left(2^{-[n / 2]} e^{-n / 2} j-1\right)^{2 / n}+\frac{1}{4} R_{\min } .
$$

Proof. From (2)

$$
j e^{-\alpha} \leq 2^{[n / 2]} e^{-\left(\alpha / 4 \lambda_{J}\right) R_{\min }}\left(1+4\left(\frac{2 c_{1}}{n} \frac{\alpha}{\lambda_{j}}\right)^{-n / 2} \operatorname{Vol}(X)\right) .
$$

Putting $\alpha=\beta \lambda_{j}$,

$$
\lambda_{j} \geq \frac{1}{\beta} \ln \left(j / 2^{[n / 2]}\right)-\frac{1}{\beta} \ln \left(1+4\left(\frac{2 C_{1}}{n}\right)^{-n / 2} \beta^{-n / 2} \operatorname{Vol}(X)\right)+\frac{1}{4} R_{\text {min }} .
$$

Thus it suffices to assume $R_{\text {min }}=0$, pick $\beta$ to estimate $\lambda_{\mathrm{j}}$ and then add $\frac{1}{4} R_{\text {min }}$. Putting $R_{\text {min }}=0$ in (3),

$$
\lambda_{j} \geq \alpha\left(\frac{1}{4} \operatorname{Vol}(X)\right)^{2 / n}\left(2 C_{1} / n\right)\left(j 2^{-[n / 2]} e^{-\alpha}-1\right)^{2 / n} .
$$

This gives lower bounds whenever $j>2^{[n / 2]}$, but to get the best power law behaviour take $\alpha=n / 2$ and $j \geq 2^{[n / 2]} e^{n / 2}$. Then

$$
\lambda_{j} \geq C_{1}(4 \operatorname{Vol}(X))^{-2 / n}\left(2^{-[n / 2]} e^{-n / 2} j-1\right)^{2 / n} .
$$

We note that $C_{1}$ can be estimated from below in terms of $\operatorname{Diam}(g)$, $\operatorname{Vol}(g)$ and $\operatorname{Ric}(g)[2]$.

\section{REFERENCES}

[1] S. -Y. Cheng, Eigenvalue comparison theorems and its geometric applications, Math. Z., 143 (1975), 289.

[2] S. -Y. Cheng and P. Li, Heat kernel estimates and lower bound of eigenvalues, Comment. Math. Helvetici, 53 (1981), 327.

[3] G. Gibbons, S. Hawkins, G. Horowitz and M. Perry, Positive mass theorems for black holes, Comm. Math. Phys., 88 (1983), 295. 
[4] N. Hitchin, Harmonic spinors, Adv. in Math., 14 (1974), 1.

[5] M. Taylor, Pseudodifferential Operators, Princeton University Press, 1981

[6] C. Vafa and E. Witten, Eigenvalue inequalities for Fermions in Gauge Theories, Princeton Univ. Physics Preprint, 1984

[7] J. Wolf, Essential self-adjointness for the Dirac operator and its square, Indiana Univ. Math. J., 22 (1972), 611.

Received May 19, 1985.

HARVARD UNIVERSITY

CAMBRIDGE, MA 02138 


\section{PACIFIC JOURNAL OF MATHEMATICS EDITORS}

\author{
V. S. VARADARAJAN \\ (Managing Editor) \\ University of California \\ Los Angeles, CA 90024 \\ HERBERT ClEMENS \\ University of Utah \\ Salt Lake City, UT 84112 \\ R. FINN \\ Stanford University \\ Stanford, CA 94305
}

\author{
HERMANN FLASCHKA \\ University of Arizona \\ Tucson, AZ 85721 \\ RAMESH A. GANGOLLI \\ University of Washington \\ Seattle, WA 98195 \\ VAUghan F. R. JONES \\ University of California \\ Berkeley, CA 94720 \\ ROBION KIRBY \\ University of California \\ Berkeley, CA 94720
}

C. C. MOORE

University of California Berkeley, CA 94720

H. SAMELSON

Stanford University Stanford, CA 94305

HAROLD STARK

University of California, San Diego La Jolla, CA 92093

\section{ASSOCIATE EDITORS}
R. ARENS
E. F. BECKENBACH
B. H. NEUMANN
F. WOLF
K. YOSHIDA (1906-1982)

\section{SUPPORTING INSTITUTIONS}

UNIVERSITY OF ARIZONA

UNIVERSITY OF BRITISH COLUMBIA

CALIFORNIA INSTITUTE OF TECHNOLOGY

UNIVERSITY OF CALIFORNIA

MONTANA STATE UNIVERSITY

UNIVERSITY OF NEVADA, RENO

NEW MEXICO STATE UNIVERSITY

OREGON STATE UNIVERSITY
UNIVERSITY OF OREGON UNIVERSITY OF SOUTHERN CALIFORNIA

STANFORD UNIVERSITY

UNIVERSITY OF HAWAII

UNIVERSITY OF TOKYO

UNIVERSITY OF UTAH

WASHINGTON STATE UNIVERSITY

UNIVERSITY OF WASHINGTON 


\section{Pacific Journal of Mathematics}

Vol. 125, No. 1 September, 1986

Gilles Christol, Fonctions et éléments algébriques $\ldots \ldots \ldots \ldots \ldots \ldots \ldots \ldots$

Jo-Ann Deborah Cohen, Extensions of valuation and absolute valued

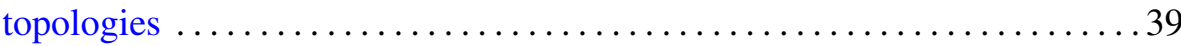

Miriam Cohen, Smash products, inner actions and quotient rings . . . . . . 45

Mikio Furushima, On the singular $K-3$ surfaces with hypersurface

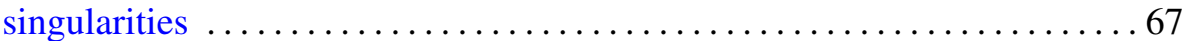

Gerhard Gierz and Boris Shekhtman, A duality principle for rational

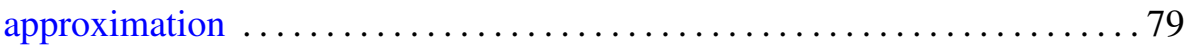

Anthony Wood Hager, A description of HSP-like classes, and

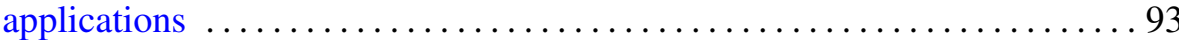

George Alan Jennings, Lines having high contact with a projective

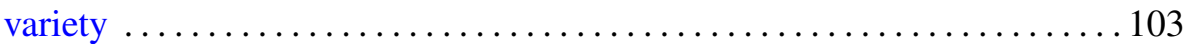

John Lott, Eigenvalue bounds for the Dirac operator . . . . . . . . . . . 117

Denis Laurent Luminet, A functional calculus for Banach PI-algebras . . . . 127

Shizuo Miyajima and Noboru Okazawa, Generators of positive

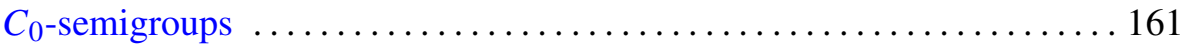

Takemi Mizokami, On functions and stratifiable $\mu$-spaces $\ldots \ldots \ldots \ldots \ldots 177$

Jeff Parker, 4-dimensional $G$-manifolds with 3-dimensional orbits . . . . . 187

Elias Saab and Paulette Saab, On Peł czyński's properties (V) and (V*) . . 205

Elmar Schrohe, The symbols of an algebra of pseudodifferential operators

Aart van Harten and Els Vader-Burger, Approximate Green functions as a tool to prove correctness of a formal approximation in a model of competing and diffusing species

Stephen Watson, Using prediction principles to construct ordered

continua 